\title{
Steady Flow of a Second-Grade Fluid in an Annulus with Porous Walls
}

\author{
M. Emin Erdoğan and C. Erdem İmrak \\ Mechanical Engineering Department, Faculty of Mechanical Engineering, Istanbul Technical University, \\ Gümüşsuyu 34437, Istanbul, Turkey
}

Correspondence should be addressed to C. Erdem İmrak, imrak@itu.edu.tr

Received 22 June 2007; Accepted 9 February 2008

Recommended by Kumbakonam Rajagopal

An exact solution of an incompressible second-grade fluid for flow between two coaxial cylinders with porous walls is given. It is assumed that the inner cylinder is rotating with a constant angular velocity and the outer one is at rest. The solution is expressed in terms of the confluent hypergeometric functions and it is valid for all values of the cross-Reynolds number and the elastic number. The solutions for $-2,+\infty$, and $-\infty$ values of the cross-Reynolds number are obtained and a comparison with those of the Newtonian fluid is given. Furthermore, the torque exerted by the fluid on the inner cylinder is calculated. It is shown that the moment coefficient depends on the cross-Reynolds number, the elastic number, and the ratio of the radii of the cylinders. The variation of the moment coefficient with these numbers is discussed.

Copyright (C) 2008 M. E. Erdoğan and C. E. İmrak. This is an open access article distributed under the Creative Commons Attribution License, which permits unrestricted use, distribution, and reproduction in any medium, provided the original work is properly cited.

\section{Introduction}

The flow considered in this paper is for flow of a second-grade fluid in an annulus with porous walls. For a Newtonian fluid, the flow in an annulus has been investigated by Berman $[1$, 2] and Yuan [3]. The extensions of this flow to non-Newtonian fluids have been studied by many authors [4-6]. However, the results for non-Newtonian fluids have been given by using a perturbation method. Therefore, it is not possible to compare with the exact solution of a Newtonian fluid for all values of the cross-Reynolds number which is defined by the suction velocity at the surface of the cylinder, the radius of the cylinder, and the kinematic viscosity of the fluid. The exact solution given in this paper provides comparison with the exact solution of a Newtonian fluid for all values of the cross-Reynolds number. The non-Newtonian fluid model in this paper is that of the second-grade fluid [7]. Although there are some criticisms on the applications of this model, many papers have been published and a listing of some of them may be found in the literature. Furthermore, it has been shown by Walters [8] that 
for many types of problems in which the flow is slow enough in the viscoelastic sense, the results given by Oldroyd's constitutive equation will be substantially similar to those of fluids of the second or third grade. Therefore, it would seem reasonable to use the second- or thirdgrade models in carrying out the calculations. This is particularly so in view of the fact that the calculation is generally simpler. The constitutive equation for an incompressible second-grade fluid has three coefficients. There are some restrictions on these coefficients due to the ClausiusDuhem inequality and due to the assumption that the Helmhotlz free energy is minimum in equilibrium. A comprehensive discussion on the restrictions for these coefficients has been given by Dunn and Fosdick [9] and Dunn and Rajagopal [10]. The sign of the material moduli is the subject of much controversy $[11,12]$.

The no-slip boundary condition is sufficient for a Newtonian fluid, but may not be sufficient for a fluid of second grade. Therefore, one needs an additional condition at boundary. However, if one uses a perturbation expansion in terms of the coefficient appearing in the higher-order derivative of the governing equation, the no-slip boundary condition is sufficient. A critical review on the boundary conditions, the existence, and uniqueness of the solution has been given by Rajagopal [13]. In order to overcome the difficulty on the boundary condition, several workers have studied to give an additional condition. Frater [14] has studied the problem without using a perturbation expansion in terms of the coefficient of the higher-order term of the governing equation. He imposed an additional condition so that the solution tends to the Newtonian value as the coefficient of the higher derivative in the equation approaches zero. This can be used provided the explicit solution or the numerical solution should exist. In this paper, the solution is obtained in terms of the confluent hypergeometric functions and has three coefficients: two of them can be determined by the no-slip boundary condition and the other can be determined by using the properties of the confluent hypergeometric functions.

The problem considered in this paper is an extension of a viscous fluid in an annulus with uniform porous walls to the problem for the flow of a second-grade fluid. The fluid injection rate at one wall is taken equal to the withdrawal rate at the outer wall. Further, because of this restriction on the cross wall, the velocity components do not vary along the axis of the cylinder. The flow in an annulus with porous walls has been investigated by many authors [4-6]. In order to obtain the solution in [4, 6], a perturbation method has been used, therefore, the results obtained can be applicable for small values of the cross-Reynolds number. The axial flow in an annulus with porous walls of a second-grade fluid has been investigated in [15]. It can be shown that the axial and the azimuthal velocities are superposable. The solution for the flow of a second-grade fluid between two parallel porous plates has been given in [16]. The flow of a second-grade fluid generated by a rotating porous cylinder has been investigated in [17]. The flow of a second-grade fluid between eccentric rotating cylinders was examined in [18]. Assuming that the eccentricity is small, the secondary flow for a third-grade fluid between two eccentrically placed cylinders has been investigated in [19]. The flow of a second-grade fluid in a porous cylindrical tube was examined for suction or injection through the surface of the tube, under the assumption that the second-order parameter is sufficiently small [20]. In this paper, the solution is obtained in terms of the confluent hypergeometric functions and it is valid for all values of the cross-Reynolds number and the elastic number. The solutions for $+\infty,-\infty$, and -2 values of the cross-Reynolds number are discussed. The solution for $+\infty$ value of the cross-Reynolds number is given in terms of the modified Bessel functions of the first kind of order one. The solution for $-\infty$ value of the cross-Reynolds number is given in terms of the modified Bessel function of the second kind of order one. The 
solution for -2 value of the cross-Reynolds number is given in terms of the exponential integral.

The torque exerted by the fluid on the inner cylinder is calculated. This can be used to obtain the values of the material coefficients. It is shown that the moment coefficient depends on the cross-Reynolds number, the elastic number, and the ratio of the radii of the cylinders. The variation of the moment coefficient with these numbers is discussed. There are appreciable differences between that of the Newtonian fluid and that of the second-grade fluid.

\section{Basic equations}

The equation of motion for a fluid in the absence of body forces is

$$
\rho \frac{D \mathbf{u}}{D t}=\nabla \cdot \sigma,
$$

where $\rho$ is the density of the fluid, $\mathbf{u}$ is the velocity, $\boldsymbol{\sigma}$ is the stress tensor, and $D / D t$ represents the material derivative. The continuity equation for the velocity is

$$
\nabla \cdot \mathbf{u}=0
$$

Equations (2.1) and (2.2) can be applied to all types of incompressible Newtonian and nonNewtonian fluids. The stress depends on the local properties of the fluid. The relation between the stress and the local properties of the fluid is called constitutive equation. The constitutive equation for an incompressible second-grade fluid is in the following form [7]:

$$
\boldsymbol{\sigma}=-p \mathbf{I}+\mu \mathbf{A}_{1}+\alpha_{1} \mathbf{A}_{2}+\alpha_{2} \mathbf{A}_{1}^{2}
$$

where $\mu, \alpha_{1}$, and $\alpha_{2}$ are material constants, and $\mathbf{A}_{n}$ represents the Rivlin-Eriksen tensor defined as [21]

$$
\begin{aligned}
& \mathbf{A}_{0}=\mathbf{I}, \quad \mathbf{A}_{1}=\nabla \mathbf{u}+(\nabla \mathbf{u})^{T} \\
& \mathbf{A}_{2}=\left(\frac{\partial}{\partial t}+\mathbf{u} \cdot \nabla\right) \mathbf{A}_{1}+\mathbf{A}_{1} \cdot(\nabla \mathbf{u})+(\nabla \mathbf{u})^{T} \cdot \mathbf{A}_{1}
\end{aligned}
$$

where $t$ is time, $p$ is pressure, and $\mathbf{I}$ is the identity tensor. The Clausius-Duhem inequality and the condition that Helmholtz free energy is minimum in equilibrium provide the following restrictions $[9,10]$ :

$$
\mu \geq 0, \quad \alpha_{1}+\alpha_{2}=0, \quad \alpha_{1} \geq 0
$$

A comprehensive discussion on the restriction for $\mu, \alpha_{1}$, and $\alpha_{2}$ can be found in the work by Dunn and Rajagopal [10]. The sign of the material moduli $\alpha_{1}$ and $\alpha_{2}$ is the subject of much controversy [11, 12]. The experiments have not confirmed these restriction on $\alpha_{1}$ and $\alpha_{2}$. Thus, the conclusion is that the fluids which have been tested are not fluids of second grade and characterized by a different constitutive structure.

Fully developed laminar flow of an incompressible fluid of second grade in an annulus with porous walls is considered. The cylindrical polar coordinates are used. The radii of the 
porous cylinders are $a_{1}$ and $b_{1}\left(a_{1}<b_{1}\right)$. The rate of fluid withdrawal at one wall of the annulus is assumed to be equal to the rate of injection of fluid at the other wall, and that these rates are independent of axial position in annulus. The condition that the suction rate at wall is equal to the injection rate at the outer is satisfied if

$$
b_{1} u_{b}=a_{1} u_{a}
$$

where $u_{a}$ and $u_{b}$ are the radial velocities at walls of smaller and larger pipes, respectively. Equation (2.6) provides that the velocity is independent of the axial position in annulus. The velocity field is assumed to be in the following form:

$$
u_{r}=\frac{\alpha}{r}, \quad u_{\theta}=u(r), \quad u_{z}=0
$$

where $u_{r}, u_{\theta}, u_{z}$ are components of the velocity in cylindrical polar coordinates, $\alpha$ is positive for injection at the inner cylinder and negative for suction at the inner one. The velocity field given by (2.7) is not a special case of the flow that is given in [22]. The velocity field in [22] is a superposition of the stagnation point flow and the rotating flow. Equation (2.2) is satisfied identically by the velocity given by (2.7). Inserting the velocity given by (2.7) into the expression of the stress, the components of the stress tensor, in cylindrical polar coordinates, can be written in the following forms:

$$
\begin{aligned}
& \sigma_{r r}=-p-\frac{2 \alpha \mu}{r^{2}}+\alpha_{1}\left[\frac{8 \alpha^{2}}{r^{4}}+2 u^{\prime 2}-2\left(\frac{u^{2}}{r}\right)^{\prime}\right]+\alpha_{2}\left[\frac{4 \alpha^{2}}{r^{4}}+\left(u^{\prime}-\frac{u}{r}\right)^{2}\right], \\
& \sigma_{r \theta}=\mu\left(u^{\prime}-\frac{u}{r}\right)+\frac{\alpha_{1} \alpha}{r}\left(u^{\prime \prime}+\frac{u^{\prime}}{r}-\frac{u}{r^{2}}\right), \\
& \sigma_{r z}=0 \\
& \sigma_{\theta \theta}=-p+2 \mu \frac{\alpha}{r^{2}}+\alpha_{2}\left[4 \frac{\alpha^{2}}{r^{4}}+\left(u^{\prime}-\frac{u}{r}\right)^{2}\right], \\
& \sigma_{\theta z}=0, \\
& \sigma_{z z}=-p,
\end{aligned}
$$

where $\sigma_{r \theta}=\sigma_{\theta r}, \sigma_{r z}=\sigma_{z r}, \sigma_{\theta z}=\sigma_{z \theta}$; the primes denote differentiation with respect to $r$. Inserting the stress components and the velocity given by (2.7) into (2.1), one obtains

$$
\alpha_{1} \alpha\left(\frac{u^{\prime \prime \prime}}{r}+2 \frac{u^{\prime \prime}}{r^{2}}-\frac{u^{\prime}}{r^{3}}+\frac{u}{r^{4}}\right)+\mu\left(u^{\prime \prime}+\frac{u^{\prime}}{r}-\frac{u}{r^{2}}\right)-\rho \frac{\alpha}{r}\left(u^{\prime}+\frac{u}{r}\right)=0
$$

and the boundary conditions are

$$
u\left(a_{1}\right)=\Omega a_{1}, \quad u\left(b_{1}\right)=0,
$$

where $a_{1}$ is the radius of the inner cylinder and $b_{1}$ is the radius of the outer cylinder. The solution to (2.9) has three coefficients: two of them can be determined by the no-slip condition (2.10) and the other can be determined by the confluent hypergeometric functions. It is 
expected that when the viscoelastic parameter goes to zero, the solution should give the Newtonian case [23], leading to entirely two different kinds of flow behavior. Similar discussions can be found in [24-26].

\section{Solution of the problem}

After some manipulations, (2.9) takes the form

$$
x y^{\prime \prime}+(1-x) y^{\prime}+\frac{R}{2} y=0,
$$

where

$$
u=\frac{g(r)}{r}, \quad g^{\prime}=\frac{r}{b_{1}^{2}} y(x), \quad x=-\frac{\xi^{2}}{2 \varepsilon R^{\prime}}, \quad \xi=\frac{r}{b_{1}}, \quad R=\frac{\alpha}{v^{\prime}}, \quad \varepsilon=\frac{\alpha_{1} / \rho}{b_{1}^{2}},
$$

where prime denotes the differentiation with respect to $r, y(x)$ is the new function, $x$ is the new variable, $R$ is the cross-Reynolds number, and $\varepsilon$ is the elastic number and the boundary conditions are

$$
g\left(a_{1}\right)=\Omega a_{1}^{2}, \quad g\left(b_{1}\right)=0 .
$$

Equation (3.3) denotes that the inner cylinder is rotating with angular velocity $\Omega$ and the outer one is at rest. The solution of (3.1) can be written in the following form:

$$
y=C_{1} M\left(-\frac{R}{2}, 1, x\right)+C_{2} U\left(-\frac{R}{2}, 1, x\right),
$$

where $M(a, b, x)$ and $U(a, b, x)$ are the confluent hypergeometric functions [27, 28]. The solution has three coefficients; therefore, one needs three boundary conditions. Thus, unless an additional condition is prescribed over the conditions (2.10), one has a parametric solution. However, the third coefficient can be determined by the properties of the confluent hypergeometric functions. For $R>0, x$ becomes negative; then for $x<0, U(a, b, x)$ is not acceptable and therefore $C_{2}$ must be zero and (3.4) takes the form

$$
\frac{d g}{d r}=C_{1} \frac{r}{b_{1}^{2}} M\left(-\frac{R}{2}, 1,-\frac{r^{2} / b_{1}^{2}}{2 \varepsilon R}\right)
$$

Using the identity in [28],

$$
M\left(-\frac{R}{2}, 1,-\frac{r^{2} / b_{1}^{2}}{2 \varepsilon R}\right)=e^{-r^{2} / b_{1}^{2} / 2 \varepsilon R} M\left(1+\frac{R}{2}, 1, \frac{r^{2} / b_{1}^{2}}{2 \varepsilon R}\right)
$$

The integration gives

$$
g=C \int e^{-z} M\left(1+\frac{R}{2}, 1, z\right) d z+D
$$


where $z=\left(r^{2} / b_{1}^{2}\right) / 2 \varepsilon R$. Using the identity in [28] which is

$$
\int e^{-z} M\left(1+\frac{R}{2}, 1, z\right) d z=z e^{-z} M\left(2+\frac{R}{2}, 2, z\right)+\text { constant }
$$

and by the boundary condition (3.3), one obtains

$$
\frac{g}{\Omega a_{1}^{2}}=\frac{\xi^{2} e^{-\xi^{2} / 2 \varepsilon R} M\left(2+R / 2,2, \xi^{2} / 2 \varepsilon R\right)-e^{-1 / 2 \varepsilon R} M(2+R / 2,2,1 / 2 \varepsilon R)}{\sigma^{2} e^{-\sigma^{2} / 2 \varepsilon R} M\left(2+R / 2,2, \sigma^{2} / 2 \varepsilon R\right)-e^{-1 / 2 \varepsilon R} M(2+R / 2,2,1 / 2 \varepsilon R)}
$$

where $u / \Omega a_{1}=(\sigma / \xi)\left(g / \Omega a_{1}^{2}\right)$ and $\sigma=a_{1} / b_{1}$. When $\varepsilon$ goes to zero, using the asymptotic expression of $M(a, b, x)$ in [28], $g / \Omega a_{1}^{2}$ becomes

$$
\lim _{\varepsilon \rightarrow 0} \frac{g}{\Omega a_{1}^{2}}=\lim _{\varepsilon \rightarrow 0} \frac{\left(r^{2} / b_{1}^{2}\right)\left(\sigma^{2} / b_{1}^{2} / 2 \varepsilon R\right)^{R / 2}-(1 / 2 \varepsilon R)^{R / 2}}{\sigma^{2}\left(\sigma^{2} / 2 \varepsilon R\right)^{R / 2}-(1 / 2 \varepsilon R)^{R / 2}}=\frac{1-\xi^{R+2}}{1-\sigma^{R+2}}
$$

or

$$
\frac{u}{\Omega a_{1}}=\frac{\sigma}{1-\sigma^{2+R}}\left(\frac{1}{\xi}-\xi^{1+R}\right)
$$

which is the expression of the velocity of a Newtonian fluid. The variation of $u / \Omega a_{1}$ with respect to $\zeta=(\xi-\sigma) /(1-\sigma)$ for various values of $R$ and $\varepsilon$ is illustrated in Figure 1. The value of $\sigma$ is taken as 0.2 and the values of $\varepsilon$ are taken as 0 and 1. Equation (3.9) is valid for all values of $R$ and $\varepsilon$. When $R$ goes to infinity, using the expression given in [28] which is

$$
\lim _{a \rightarrow \infty} \frac{1}{\Gamma(b)} M\left(a, b, \frac{x}{a}\right)=x^{1 / 2-(1 / 2) b} I_{b-1}(2 \sqrt{x})
$$

equation (3.9) takes the following form:

$$
\frac{u}{\Omega a_{1}}=\frac{\sigma}{\xi} \frac{\xi I_{1}\left(\varepsilon^{-1 / 2} \xi\right)-I_{1}\left(\varepsilon^{-1 / 2}\right)}{\sigma I_{1}\left(\varepsilon^{-1 / 2} \sigma\right)-I_{1}\left(\varepsilon^{-1 / 2}\right)}
$$

where $\Gamma(x)$ is the Gamma function and $I_{1}(x)$ is the modified Bessel function of the first kind of order one. The variation of $u / \Omega a_{1}$ with respect to $\zeta$ for various values of $\varepsilon$ is illustrated in Figure 1. Since the asymptotic form of $I_{1}(x)$ is $e^{x} / \sqrt{2 \pi x}$ when $\varepsilon$ goes to zero, (3.13) becomes

$$
\frac{u}{\Omega a_{1}}=\frac{\sigma}{\xi} .
$$




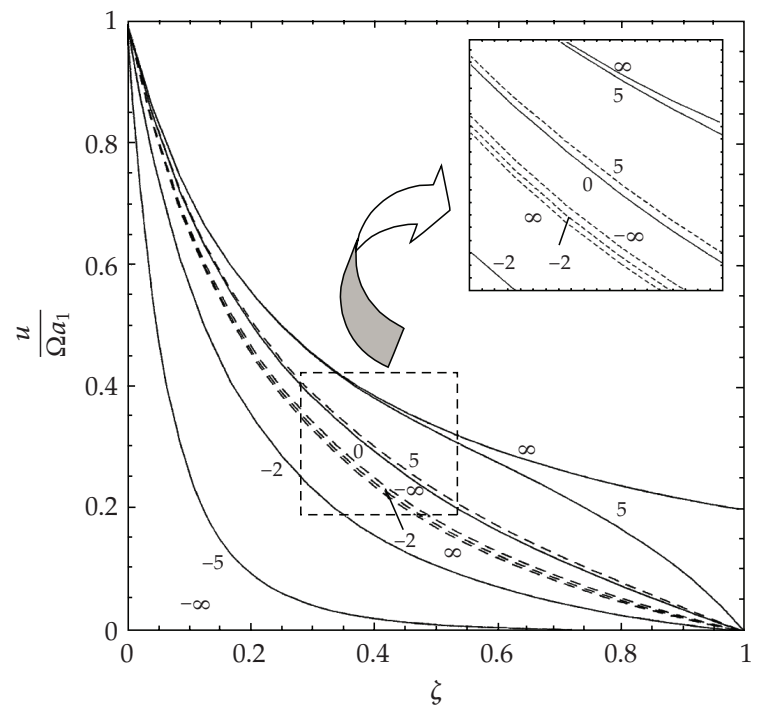

Figure 1: The variation of the azimuthal velocity for various values of the cross-Reynolds number and the elastic number. $(\varepsilon=0) ;(\varepsilon=1), \zeta=(\xi-\sigma) /(1-\sigma) ; \sigma \leq \xi \leq r / b_{1}, \sigma=0.2$.

Equation (3.14) satisfies the boundary condition at $\xi=\sigma$, but it does not satisfy the boundary condition at $\xi=1$. It is clearly seen from Figure 1 that when $R$ goes to $+\infty$, the flow shows a boundary layer character near the outer cylinder for a Newtonian fluid, but it does not behave the same way for a fluid of second grade. A similar situation occurs for the axial flow between two circular cylinders with porous walls of a second-grade fluid [15].

For $R<0, x$ is positive; then for $x>0, M(a, b, x)$ becomes an increasing function of $x$; therefore, $C_{1}$ must be zero, then (3.4) takes the form

$$
\frac{d g}{d r}=C_{2} \frac{r}{b_{1}^{2}}\left(\frac{N}{2}, 1, \frac{r^{2} / b_{1}^{2}}{2 \varepsilon N}\right)
$$

where $N=-R(>0)$. Using the identity in [28] which is

$$
\int U(a, 1, z) d z=\frac{1}{1-a} U(a-1,0, z)+\text { constant }
$$

and by the boundary condition, the integration gives

$$
\frac{g}{\Omega a_{1}^{2}}=\frac{U\left(N / 2-1,0, r^{2} / b_{1}^{2} / 2 \varepsilon R\right)-U(N / 2-1,0,1 / 2 \varepsilon N)}{U\left(N / 2-1,0, \sigma^{2} / 2 \varepsilon N\right)-U(N / 2-1,0,1 / 2 \varepsilon N)},
$$

where $u / \Omega a_{1}=(\sigma / \xi)\left(g / \Omega a_{1}^{2}\right)$. When $\varepsilon$ goes to zero, using the asymptotic expression of $U(a, b, x)$ in [28], one obtains

$$
\frac{u}{\Omega a_{1}}=\frac{\sigma}{1-\sigma^{2-N}}\left(\frac{1}{\xi}-\xi^{1-N}\right)
$$


which is the expression of the velocity for a Newtonian fluid. The variation of $u / \Omega a_{1}$, with respect to $\zeta=(\xi-\sigma) /(1-\sigma)$ for various values of $-R$ and $\varepsilon$, is illustrated in Figure 1 . The value of $\sigma$ is taken as 0.2 and the values of $\varepsilon$ are taken as 0 and 1. Equation (3.17) is valid for all values of $-R$ and $\varepsilon$. When $N$ goes to infinity, using the asymptotic expression given in [28] which is

$$
\lim _{a \rightarrow \infty} \Gamma(1+a-b) \mathrm{U}\left(a, b, \frac{x}{a}\right)=2 x^{1 / 2-(1 / 2) b} K_{b-1}(2 \sqrt{x}) .
$$

Equation (3.17) takes the following form:

$$
\frac{u}{\Omega a_{1}}=\frac{\sigma}{\xi} \frac{\xi K_{1}\left(\varepsilon^{-1 / 2} \xi\right)-K_{1}\left(\varepsilon^{-1 / 2}\right)}{\sigma K_{1}\left(\varepsilon^{-1 / 2} \sigma\right)-K_{1}\left(\varepsilon^{-1 / 2}\right)},
$$

where $K_{1}(x)$ is the modified Bessel function of the second kind of order one. The variation of $u / \Omega a_{1}$ with respect to $\zeta$ for various values of $\varepsilon$ is illustrated in Figure 1 . Since the asymptotic form of $K_{1}(x)$ is $\sqrt{\pi / 2 x} e^{-x}$ when $\varepsilon$ goes to zero, (3.20) becomes

$$
\frac{u}{\Omega a_{1}}=0
$$

Equation (3.21) satisfies the boundary condition at $\xi=1$, but it does not satisfy the boundary condition at $\xi=\sigma$. The reason is that a boundary layer occurs at $\xi=\sigma$ for a Newtonian fluid, but it does not behave the same way for a fluid of second grade.

The case of $R=-2$, namely $N=2$, cannot be obtained by (3.17). For $N=2$, by the definition of $U(a, b, x), U(1,1, x)$ can be found as

$$
U(1,1, x)=e^{x} \operatorname{Ei}(x)
$$

where $\operatorname{Ei}(x)$ is the exponential integral defined in the following form

$$
E i(x)=\int_{x}^{\infty} \frac{e^{-t}}{t} d t
$$

Inserting $U(1,1, x)$ into the expression of $d g / d r$ and by integration and by using the boundary conditions, one finds

$$
\frac{u}{\Omega a_{1}}=\frac{\sigma}{\xi} \frac{e^{\xi^{2} / 4 \varepsilon} E i\left(\xi^{2} / 4 \varepsilon\right)-e^{1 / 4 \varepsilon} E i(1 / 4 \varepsilon)+\ln \left(\xi^{2}\right)}{e^{\sigma^{2} / 4 \varepsilon} E i\left(\sigma^{2} / 4 \varepsilon\right)-e^{1 / 4 \varepsilon} \operatorname{Ei}(1 / 4 \varepsilon)+\ln \left(\sigma^{2}\right)} .
$$

The variation of $\Omega a_{1}$ with respect to $\zeta$ for various values of $\varepsilon$ is illustrated in Figure 1 . The value of $\sigma$ is taken as 0.2 and the values of $\varepsilon$ are taken as 0 and 1 . When $\varepsilon$ goes to zero, the argument of the exponential integral becomes large, then $E i(x)$ can be written as $e^{-x} / x$ and (3.24) becomes

$$
\frac{u}{\Omega a_{1}}=\frac{\sigma}{\xi} \frac{\ln \xi}{\ln \sigma}
$$




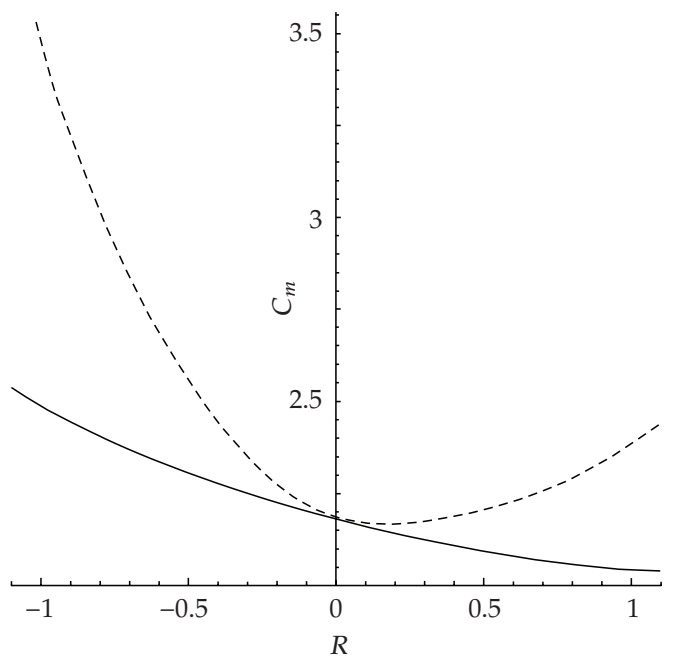

Figure 2: The variation of the moment coefficient with the cross-Reynolds number. The ratio of the radii of the cylinders is taken as 0.2. $(\varepsilon=0) ;(\varepsilon=1)$,

\section{The torque exerted by the fluid on the inner cylinder}

The torque exerted by the flow on the inner cylinder is given in the following form:

$$
M=2 \pi h a_{1}^{2}\left[\mu\left(\frac{d u}{d r}-\frac{u}{r}\right)+\frac{\alpha \alpha_{1}}{r} \frac{d}{d r}\left(\frac{d u}{d r}+\frac{u}{r}\right)\right]_{r=a_{1}},
$$

where $h$ is the length of the inner cylinder. The moment coefficient is

$$
\left(C_{m}\right)_{r=a_{1}}=\sigma\left[\left(\frac{d u}{d \xi}-\frac{u}{\xi}\right)+\frac{\varepsilon R}{\xi} \frac{d}{d \xi}\left(\frac{d u}{d \xi}+\frac{u}{\xi}\right)\right]_{\xi=\sigma} .
$$

Equation (4.2) shows that the moment coefficient depends on the cross-Reynolds number, the elastic number, and the ratio of the radii of the cylinders. The variation of the moment coefficient with these numbers is illustrated in Figure 2. There are appreciable differences between that of the Newtonian fluid and that of the second-grade fluid. The curves in Figure 2 show the effect of the second-grade fluid. The moment coefficient decreases with the cross-Reynolds number for a Newtonian fluid and increases with the cross-Reynolds number for a secondgrade fluid, for all values of the ratio of the radii of the cylinders.

\section{Conclusions}

An exact solution of the governing equation for the flow of a fluid of second grade between two coaxial cylinders with porous walls is given. The flow of a second-grade fluid for which the inner cylinder is rotating and the outer one is at rest is considered. The solution is given in terms of the confluent hypergeometric functions and is valid for all values of the cross-Reynolds number and the elastic parameter. The boundary conditions are the no-slip boundary conditions and an additional boundary condition. The additional boundary condition is that the 
solution tends to the Newtonian value as the elastic number in the solution approaches zero. The solution is discussed for positive, negative, infinite, and -2 values of the cross-Reynolds number. The torque exerted by the fluid on the inner cylinder is calculated. It is shown that the moment coefficient depends on the cross-Reynolds number, the elastic number, and the ratio of the radii of the cylinders. For a given value of this ratio, it decreases with the crossReynolds number for a Newtonian fluid and increases with the cross-Reynolds number for a second-grade fluid.

\section{Acknowledgments}

It is pleasure to acknowledge many stimulating correspondences with Professor K. R. Rajagopal and the authors would like to express their thanks to the referees for the valuable suggestions.

\section{References}

[1] A. S. Berman, "Laminar flow in channels with porous walls," Journal of Applied Physics, vol. 24, no. 9 , pp. $1232-1235,1953$.

[2] A. S. Berman, "Laminar flow in an annulus with porous walls," Journal of Applied Physics, vol. 29, no. 1, pp. 71-75, 1958.

[3] S. W. Yaun, "Further investigation of laminar flow in channels with porous walls," Journal of Applied Physics, vol. 27, no. 3, pp. 267-269, 1956.

[4] J. N. Kapur and S. Goel, "Flow of a non-Newtonian fluid between rotating cylinders with suction and injection," Physics of Fluids, vol. 6, no. 5, pp. 626-631, 1963.

[5] S. P. Mishra and J. S. Roy, "Laminar elasticoviscous flow in an annulus with porous walls," Physics of Fluids, vol. 10, no. 11, pp. 2300-2304, 1967.

[6] S. P. Mishra and J. S. Roy, "Flow of elasticoviscous liquid between rotating cylinders with suction and injection," Physics of Fluids, vol. 11, no. 10, pp. 2074-2081, 1968.

[7] K. R. Rajagopal and P. N. Kaloni, "Some remarks on boundary conditions for flows of fluids of the differential type," in Continuum Mechanics and Its Applications, G. A. C. Graham and S. K. Malik, Eds., pp. 935-942, Hemisphere, New York, NY, USA, 1989.

[8] K. Walters, "Relation between Coleman-Noll, Rivlin-Ericksen, Green-Rivlin and Oldroyd fluids," Zeitschrift für Angewandte Mathematik und Physik, vol. 21, no. 4, pp. 592-600, 1970.

[9] J. E. Dunn and R. L. Fosdick, "Thermodynamics, stability, and boundedness of fluids of complexity 2 and fluids of second grade," Archive for Rational Mechanics and Analysis, vol. 56, no. 3, pp. 191-252, 1974.

[10] J. E. Dunn and K. R. Rajagopal, "Fluids of differential type: critical review and thermodynamic analysis," International Journal of Engineering Science, vol. 33, no. 5, pp. 689-729, 1995.

[11] K. R. Rajagopal, "Flow of viscoelastic fluids between rotating disks," Theoretical and Computational Fluid Dynamics, vol. 3, no. 4, pp. 185-206, 1992.

[12] R. L. Fosdick and K. R. Rajagopal, "Anomalous features in the model of "second order fluids", " Archive for Rational Mechanics and Analysis, vol. 70, no. 2, pp. 145-152, 1979.

[13] K. R. Rajagopal, "On boundary conditions for fluids of the differential type," in Navier-Stokes Equations and Related Nonlinear Problems, A. Sequiera, Ed., pp. 273-278, Plenum Press, New York, NY, USA, 1995.

[14] K. R. Frater, "On the solution of some boundary value problems arising in elastico-viscous fluid mechanics," Zeitschrift für Angewandte Mathematik und Physik, vol. 21, no. 1, pp. 134-137, 1970.

[15] M. E. Erdoğan and C. E. İmrak, "On the steady flow of a second-grade fluid between two coaxial porous cylinders," Mathematical Problems in Engineering, vol. 2007, Article ID 42651, 11 pages, 2007.

[16] M. E. Erdoğan and C. E. İmrak, "The effects of the side walls on the flow of a second grade fluid in ducts with suction and injection," International Journal of Non-Linear Mechanics, vol. 42, no. 5, pp. 765-772, 2007.

[17] N. Roy, "Steady circulatory flow about the rotating circular cylinder with uniformly distributed suction at the surface," International Journal of Non-Linear Mechanics, vol. 19, no. 3, pp. 235-243, 1984. 
[18] I. Christie, K. R. Rajagopal, and A. Z. Szeri, "Flow of a non-Newtonian fluid between eccentric rotating cylinders," International Journal of Engineering Science, vol. 25, no. 8, pp. 1029-1047, 1987.

[19] F. Mollica and K. R. Rajagopal, "Secondary flows due to axial shearing of a third grade fluid between two eccentrically placed cylinders," International Journal of Engineering Science, vol. 37, no. 4, pp. 411$429,1999$.

[20] S. Özer and E. S. Şuhubi, "Flow of a second grade fluid through a cylindrical permeable tube," Applied Mathematics and Computation, vol. 179, no. 2, pp. 672-687, 2006.

[21] R. S. Rivlin and J. L. Ericksen, "Stress-deformation relations for isotropic materials," Journal of Rational Mechanics and Analysis, vol. 4, pp. 323-425, 1955.

[22] M. E. Erdoğan and C. E. İmrak, "An exact solution of the governing equation of a fluid of secondgrade for three-dimensional vortex flow," International Journal of Engineering Science, vol. 43, no. 8-9, pp. 721-729, 2005.

[23] M. M. Denn and K. C. Penteous, "Elastic effect in flow of viscoelastic liquids," Chemical Engineering Journal, vol. 2, no. 4, pp. 280-286, 1971.

[24] K. R. Rajagopal and A. S. Gupta, "An exact solution for the flow of a non-Newtonian fluid past an infinite porous plate," Meccanica, vol. 19, no. 2, pp. 158-160, 1984.

[25] R. L. Fosdick and B. Bernstein, "Nonuniqueness of second-order fluids under steady radial flow in annuli," International Journal of Engineering Science, vol. 7, no. 6, pp. 555-569, 1969.

[26] T. N. G. Abbott and K. Waltes, "Rheometrical flow systems part 2. Theory for the orthogonal rheometer, including an exact solution of the Navier-Stokes equations," Journal of Fluid Mechanics, vol. 40, no. 1, pp. 205-213, 1970.

[27] L. J. Slater, Confluent Hypergeometric Functions, Cambridge University Press, New York, NY, USA, 1960.

[28] M. Abromowitz and I. A. Stegun, Handbook of Mathematical Functions, New York, NY, USA, Dover, 1965. 


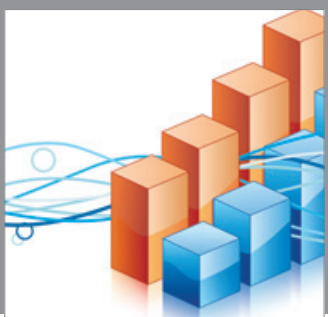

Advances in

Operations Research

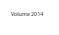

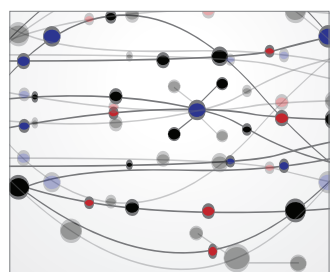

\section{The Scientific} World Journal
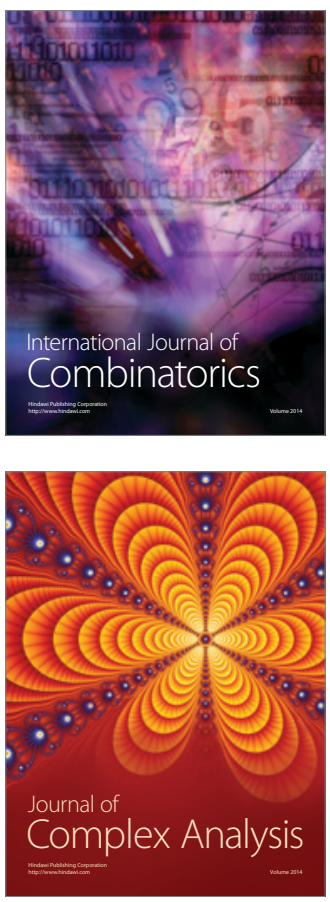

International Journal of

Mathematics and

Mathematical

Sciences
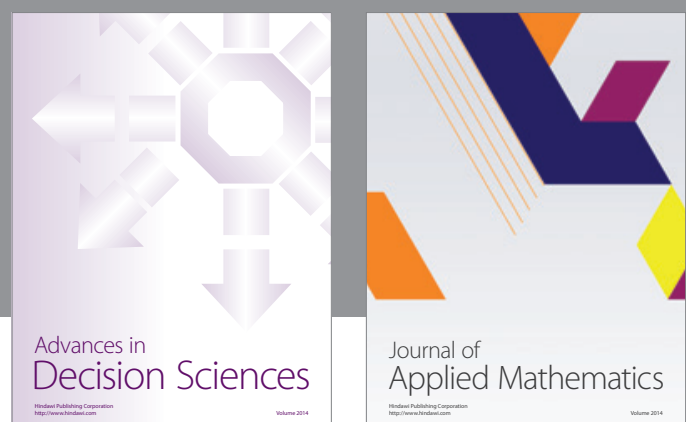

Journal of

Applied Mathematics
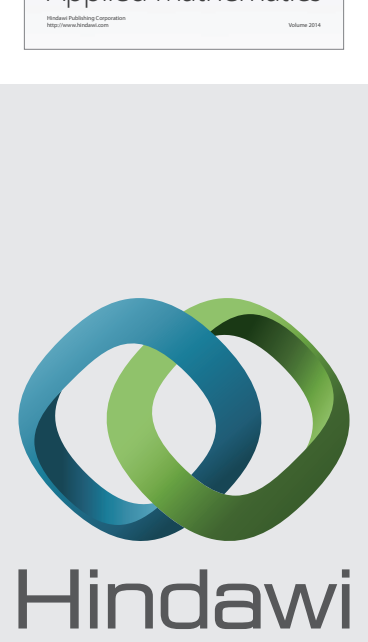

Submit your manuscripts at http://www.hindawi.com
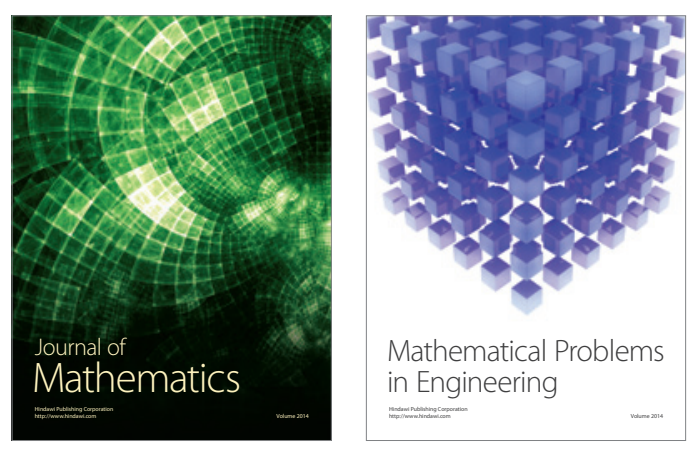

Mathematical Problems in Engineering
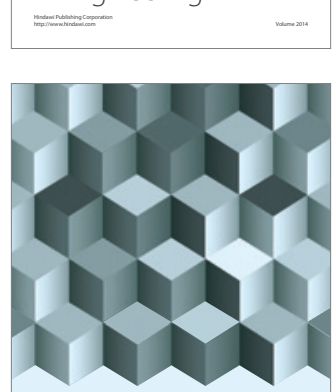

Journal of

Function Spaces
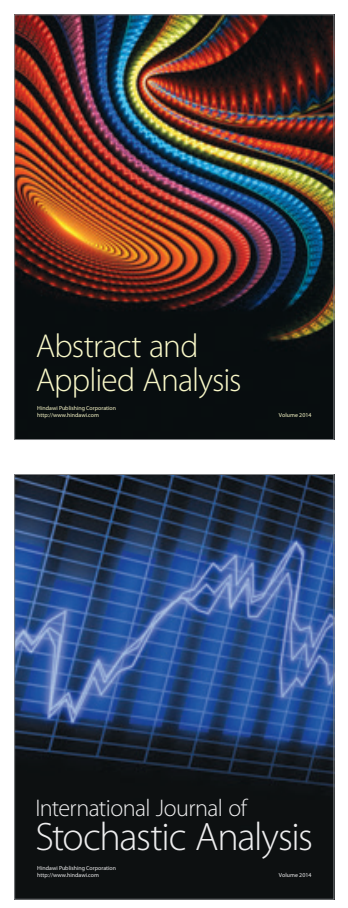

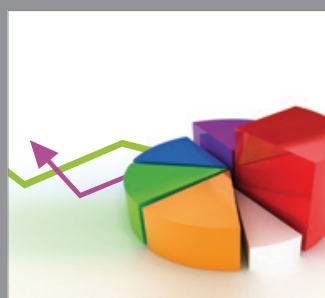

ournal of

Probability and Statistics

Promensencen
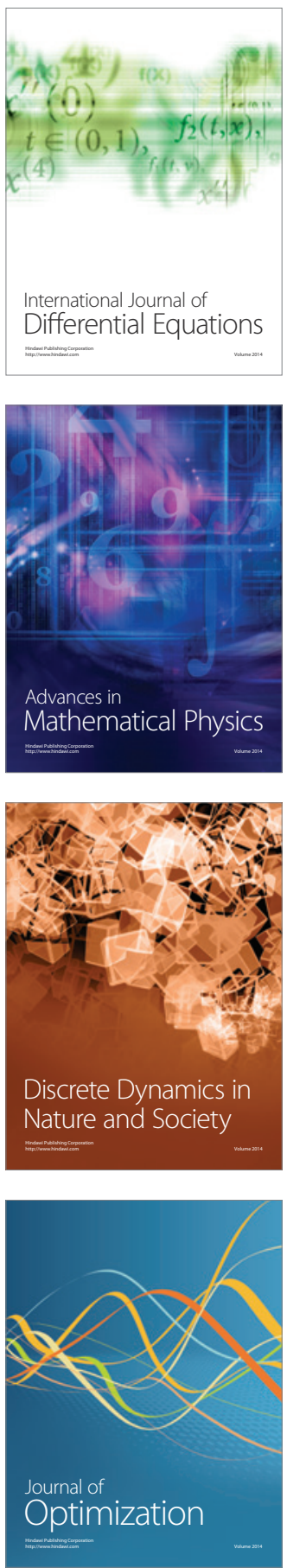